\title{
Critical wavelength for river meandering
}

\author{
Boyd F. Edwards* and Duane H. Smith \\ National Energy Technology Laboratory, U. S. Department of Energy, 3610 Collins Ferry Road, Morgantown, West Virginia 26507-0880
}

(Received 1 May 2000; published 28 March 2001)

\begin{abstract}
A fully nonlinear modal analysis identifies a critical centerline wave number $q_{c}$ for river meandering that separates long-wavelength bends, which grow to cutoff, from short-wavelength bends, which decay. Exact, numerical, and approximate analytical results for $q_{c}$ rely on the Ikeda, Parker, and Sawai [J. Fluid Mech. 112, 363 (1981)] model, supplemented by dynamical equations that govern the river migration and length. Predictions also include upvalley bend migration at long times and a peak in lateral migration rates at intermediate times. Experimental tests are suggested.
\end{abstract}

DOI: 10.1103/PhysRevE.63.045304

PACS number(s): 47.54.+r, 92.40.Fb, 47.20.-k

Among the most fascinating behaviors of alluvial rivers is their tendency to meander and rework their floodplains. Some meander bends of the lower Mississippi move $20 \mathrm{~m}$ laterally per year [1]. Even on a planar floodplain, river bends grow slowly in amplitude and accordingly increase the river sinuosity $S=L / L_{0}$, defined as the ratio of the total river length $L$ to the linear distance $L_{0}$ between its endpoints. As the sinuosity increases, adjacent river bends occasionally meet each other and cut off an oxbow lake, thereby suddenly shortening the river and reducing its sinuosity.

Rivers meander because large downstream velocities erode soil from the outside banks of large-wavelength river bends [2], causing such bends to grow in amplitude. Shortwavelength bends decay because Bernoulli's principle demands large velocities and erosion at their inside banks. Bends migrate down-valley because the cross-stream shear in the downstream velocity requires a downstream distance $D=H / 2 C_{f}$ to recover from changes in the channel curvature [3]. Here, $H$ is the average depth and $C_{f}$ is a dimensionless friction coefficient.

Natural rivers are fully developed turbulent boundary layers, with large typical Reynolds numbers $\operatorname{Re}=U H / \nu \approx 10^{6}$, where $U$ is the average downstream velocity and $\nu$ is the kinematic viscosity of water. Consequently, momentum transport is dominated by stretching and folding of turbulent eddies, rather than by viscous diffusion. To close the system of equations, the turbulent downstream bed stress $\tau_{s}$ $=\rho C_{f} U^{2}$ is evaluated using constant $C_{f}$ and constant mass density $\rho$. In mechanical equilibrium, the upstream bed force per unit area $\tau_{s}$ on the overlying fluid volume must balance the downstream component of gravitational force per unit area $\rho g H I$, whence [2] $U=\left(g H I / C_{f}\right)^{1 / 2}$, where $g$ is the acceleration of gravity and $I$ is the downstream bedslope. A dimensionless bank erodibility $E$ is defined as the proportionality constant between the normal bank migration rate and the excess near-bank downstream fluid velocity [2]. Increasing the sinuosity of a river of constant width $2 b$ and constant discharge $Q=2 b H U$ lowers its average slope $I$ $=I_{0} / S$ and its flow velocity $U=U_{0} S^{-1 / 3}$, and raises its depth $H=H_{0} S^{1 / 3}$, where $I_{0}, U_{0}$, and $H_{0}$ are values for a

\footnotetext{
*Permanent address: Department of Physics, West Virginia University, Morgantown, WV 26506-6315.
}

straight river between the same initial and final points as the sinuous river. Because of these sinuosity dependencies, dimensionless variables used henceforth employ the straightened length and time scales $D_{0}=H_{0} / 2 C_{f}$ and $T_{0}$ $=D_{0}^{2} / b E U_{0}$.

In freely meandering rivers, bed erosion at humps and deposition at hollows produce a uniform downstream bed slope $I$, apart from small-scale dunes and ripples. The corresponding linear centerline bed elevation $z=z_{0}-I s$ involves the downstream distance $s$ measured along the bed centerline. The horizontal projection of the river is conveniently described at time $t$ by the angle $\theta(s, t)$ between the downstream direction and the fixed cartesian direction $\hat{x}$. The corresponding channel curvature $\kappa(s, t)=-\partial \theta / \partial s$, measured as positive for turns to the right, must respond to the normal migration velocity $v(s, t)$, measured as positive for channel migration to the left, as seen by a local riverbound observer facing downstream.

The Ikeda, Parker, and Sawai equation [2] predicts the curvature-dependent migration velocity for quasisteady nonresonant turbulent flow in shallow sinuous channels. It is convenient to rewrite this equation using dimensionless variables [3],

$$
S^{1 / 3} \frac{\partial v}{-\partial s}+v=\frac{\partial \kappa}{\partial s}+\frac{\mathcal{P}}{S^{1 / 3}} \kappa .
$$

Here the dimensionless number $\mathcal{P}$, which we call the ' $P a r k e r$ number', in honor of Parker's pioneering contributions, measures the importance of the secondary flow. From right to left, the terms in Eq. (1) account for the secondary flow, the free shear, the downstream decay of cross-stream shear, and the resulting changes in the migration rate. The typical value $\mathcal{P} \approx 5$ includes the transport of downstream momentum by the secondary flow discussed by Johannesson and Parker [4]. To track the centerline motion, most previous studies of the Ikeda, Parker, and Sawai equation rely on coordinate transformations [2,5-7] or on numerical implementations [8-10].

To track the centerline motion, we employ exact dimensionless nonlinear dynamical equations of motion governing the river length and shape,

$$
\frac{d L}{d t}=\int_{0}^{L} \kappa v d s-u_{0}+u_{L},
$$




$$
\frac{\partial \theta}{\partial t}=\frac{\partial v}{\partial s}+\kappa \int_{0}^{s} \kappa\left(s^{\prime}, t\right) v\left(s^{\prime}, t\right) d s^{\prime}-\kappa u_{0},
$$

which may be derived [3] from Eq. (4) of Ref. [11], and which include river stretching and shrinking through the nonlinear integral terms. Reference [11] treats stretching and shrinking exactly, but replaces Eq. (1) by a local relationship which precludes down-valley migration of meander bends, an essential feature of real rivers. To allow for maximum flexibility in defining the upstream $(s=0)$ and downstream $(s=L)$ ends of the river, Eqs. (2) and (3) include the respective downstream migration velocities $u_{0}$ and $u_{L}$. Equation (3), with $u_{0}=0$, appears in previous studies of periodic rivers [12,13]. Equation (2), introduced here, allows explicit investigation of the time evolution of the river length, and plays a key role in the derivation of our fully nonlinear stability results.

Equation (2) gives the time rate of change of the river length, including both lateral and downstream contributions. During a time $d t$, river migration displaces the centerline laterally a distance $v d t$, stretching an infinitesimal centerline arc of length $d s$ and radius $R$ to a new length $d s^{\prime}$ and radius $R^{\prime}=R+v d t$, while the subtended angle $d s / R=d s^{\prime} / d R^{\prime}$ remains unchanged. Integrating the resulting relation $d s^{\prime}=(1$ $+\kappa v d t) d s$ gives the lateral contribution $\int_{0}^{L} \kappa v d s$ to Eq. (2), where $\kappa=1 / R$ is the local curvature. A downstream contribution $u_{L}-u_{0}$ is also included to allow flexibility in defining the ends of the river.

To study the nonlinear dynamics of periodic meander patterns, we write $u_{0}=u_{L}$ and

$$
\theta(s, t)=\sum_{l=-\infty}^{+\infty} \theta_{l}(t) e^{i l q s}
$$

with a time-dependent centerline wave number $q=2 \pi / L$ and wavelength $L$, fixed Cartesian wave number $q_{0}=2 \pi / L_{0}$ and wavelength $L_{0}$, sinuosity $S=L / L_{0}=q_{0} / q$, and the reality condition $\theta_{-l}=\theta_{l}^{*}$. Accordingly, Eqs. (1)-(3) reduce to

$$
\begin{aligned}
\frac{d S}{d t} & =-2 q^{2} S^{2 / 3} \sum_{l=1}^{\infty} \frac{l^{2} q^{2}-\mathcal{P} S^{-2 / 3}}{l^{2} q^{2}+S^{-2 / 3}} l^{2}\left|\theta_{l}\right|^{2}, \\
\frac{d \theta_{l}}{d t}= & \left(l^{2} q^{2} A_{l}+i l q u_{0}\right) \theta_{l}-q^{2} \sum_{\substack{m, n=-\infty \\
m \neq n}}^{+\infty} \frac{m n}{m-n} A_{n} \\
& \times\left[l \theta_{l}-(l+m-n) \theta_{l+m-n}\right] \theta_{-m} \theta_{n},
\end{aligned}
$$

with $A_{l}=\left(\mathcal{P} S^{-1 / 3}-i l q\right) /\left(1+i l q S^{1 / 3}\right)$.

Linearizing Eqs. (5) for small $\theta_{ \pm 1}=\mp(i \epsilon / 2) e^{\sigma t} e^{\mp i \omega t}$ with $u_{0}=0$ recovers the frequency $\omega=q^{3}(1+\mathcal{P}) /\left(1+q^{2}\right)$, growth rate $\sigma=q^{2}\left(\mathcal{P}-q^{2}\right) /\left(1+q^{2}\right)$, sinuosity $S=1$, and down-valley migration rate $c=\omega / q$ of small-amplitude perturbations about a straight river [2,3]. This growth rate peaks at $q=q_{m}=\left[-1+(1+\mathcal{P})^{1 / 2}\right]^{1 / 2}$ and is negative for $q>q_{c}$ $=\mathcal{P}^{1 / 2}$. The wavelength $\lambda_{m}=2 \pi D_{0} / q_{m}$ of maximum growth (in conventional units) sets the scale for alluvial meanders

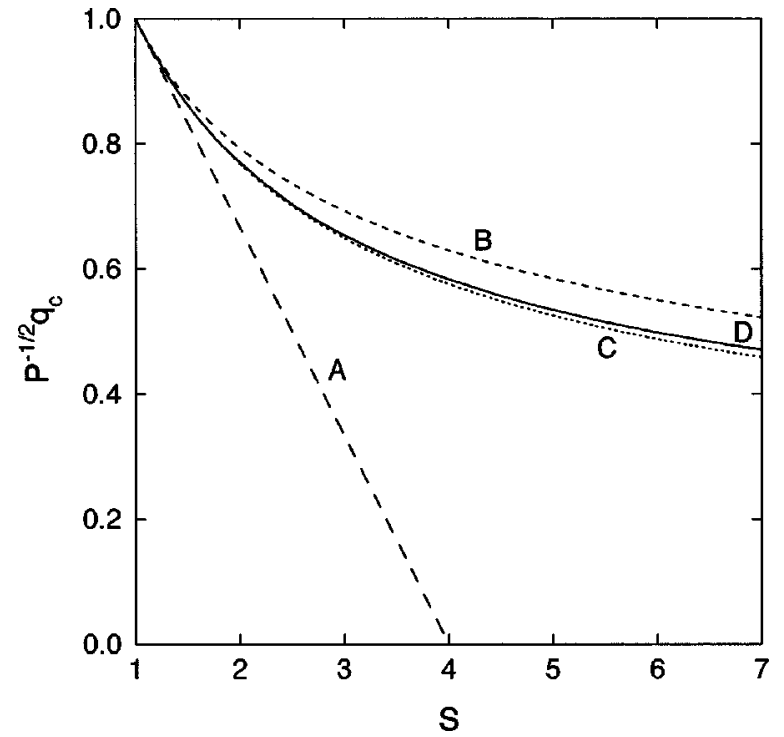

FIG. 1. Exact and approximate nonlinear results for the critical dimensionless centerline wave number $q_{c}$ vs the river sinuosity $S$ $=L / L_{0}$. Here, the "centerline wavelength" $L$ is the distance between adjacent bend apexes measured along the river centerline, and the "cartesian wavelength" $L_{0}$ is this distance measured along a straight line. At the globally unstable fixed point $q=2 \pi / L=q_{c}$, finite-amplitude periodic meander patterns migrate downvalley at constant speed without changing form. Small-wavelength bends with $q>q_{c}$ are obliterated by river straightening, whereas largewavelength bends with $q<q_{c}$ grow unrestrained until adjacent bends meet and abandon an oxbow lake. Thus, oxbow cutoff is the predicted fate of growing meander bends unhindered by natural topography or by human intervention. Since small-wavelength bends quickly disappear, large-wavelength bends are predicted to dominate in nature. An expansion to third order in the bend amplitude [5,7] gives $\mathcal{P}^{-1 / 2} q_{c}=(4-S) / 3$ [trace $A$, Eq. (7)], valid for $S$ $\rightarrow 1$, where $\mathcal{P}$ measures the importance of the secondary flow. The exact upper limit $\mathcal{P}^{-1 / 2} q_{c}=S^{-1 / 3}$ [trace $B$, Eq. (8)] holds for arbitrary $S$ and for arbitrary periodic rivers; bends with $q>\mathcal{P}^{1 / 2} S^{-1 / 3}$ are obliterated by straightening regardless of the details of their Fourier spectra. Equation (9) supplies an explicit analytical estimate [trace $C$, for $\mathcal{P}=5$ ] which agrees with exact numerical results [trace $D$ (solid line), for $\mathcal{P}=5$ ] to within $3 \%$ for $S \leqslant 7$.

observed in numerous natural and experimental rivers, from 10 -cm-wide laboratory flumes to the 1-km-wide Mississippi $[2,14]$.

A finite-amplitude solution [6] that travels down-valley without changing form follows from Eqs. (5) by setting $d \theta_{l} / d t=0$ and $u_{0}=\left.c \cos \theta\right|_{s=0}$, and by expanding to third order in the amplitude $\epsilon$ with $\theta_{ \pm 1}^{(1)}=\mp i \epsilon / 2$. The third-order contribution $\theta_{ \pm 3}^{(3)}=\left(\mathcal{P}^{1 / 2} \mp i / 3\right) \epsilon^{3} / 128$ to the resulting $\mathrm{Ki}$ noshita curve [3],

$$
\theta(s)=\epsilon \sin (q s)+\frac{\epsilon^{3}}{64}\left(\mathcal{P}^{1 / 2} \cos 3 q s+\frac{1}{3} \sin 3 q s\right),
$$

predicts bend skewing similar to that found in nature [5]. Combining the associated relations $S=1+\epsilon^{2} / 4$ and $q=q_{c}$ $=\mathcal{P}^{1 / 2}\left(1-\epsilon^{2} / 12\right)$ gives (Fig. 1, trace $\left.A\right)$ 


$$
q_{c}=\frac{\mathcal{P}^{1 / 2}}{3}(4-S),
$$

which agrees with the linear result $q_{c}=\mathcal{P}^{1 / 2}$ at $S=1$. This third-order solution is unstable [5]; that is, $q>q_{c}$ leads eventually to river straightening and $q<q_{c}$ leads to cutoff.

Equation (5a) readily yields an exact upper limit on $q_{c}$ in the fully nonlinear regime. For $q>\mathcal{P}^{1 / 2} S^{-1 / 3}$ initially, all modes make negative contributions to $d S / d t$ for all later times because $q_{0}=q S$ is fixed. Accordingly, an arbitrary finite-amplitude river whose centerline wave number $q$ exceeds the value (Fig. 1, trace $B$ )

$$
q_{c}=\frac{\mathcal{P}^{1 / 2}}{S^{1 / 3}}
$$

must straighten with time. This is an exact nonlinear result, valid for arbitrary periodic river shapes, being independent of the relative values of the mode amplitudes $\theta_{l}$. A firstorder Taylor expansion of Eq. (8) about $S=1$ recovers Eq. (7).

If $q<\mathcal{P}^{1 / 2} S^{-1 / 3}$ initially, at least the $l=1$ mode contributes positively to $d S / d t$, while the remaining modes contribute negatively. To account approximately for this competition, we insert the amplitude-expansion results $\theta_{1}^{(1)}$ and $\theta_{3}^{(3)}$ into Eq. (5a), set $d S / d t=0$ and $q=q_{c}=\mathcal{P}^{1 / 2} S^{-1 / 3}(1-\delta)$, and ignore the small correction $\delta$ except in the numerator of the $l=1$ term, which yields (Fig. 1 , trace $C$ )

$$
q_{c}=\frac{\mathcal{P}^{1 / 2}}{S^{1 / 3}}\left(1-\frac{1+\mathcal{P}}{1024} \epsilon^{4}\right) .
$$

We can render Eq. (9) an explicit function of $S$ by ignoring third-order effects in the small correction term and by thereby employing the explicit series result [3]

$$
\frac{1}{S}=1-\frac{\epsilon^{2}}{4}+\frac{\epsilon^{4}}{64}-\frac{\epsilon^{6}}{2304}+\cdots
$$

for the "sine-generated curve" [15] $\theta=\epsilon \sin q s$. Inversion using only those terms shown gives the needed relation $\epsilon^{2}$ $=12\left(1-A_{+}+A_{-}\right)$, where $A_{ \pm}^{3}=\left(\alpha^{2}+1 / 27\right)^{1 / 2} \pm \alpha$ and $\alpha$ $=(1+2 / S) / 3$. This gives $\epsilon$ to within $0.2 \%$ for $S \leqslant 7$.

Numerical integration [3] of Eqs. (5) yields further insights. To determine $u_{0}$, we assign the $s=0$ point to a bend apex where $\theta(0, t)=\Sigma_{l=-\infty}^{+\infty} \theta_{l}(t)=0$ for all time, so that $u_{0}$ and $v_{0}$ respectively represent the time-dependent components of the velocity of this apex in the $x$ (down-valley) and $y$ (cross-valley) directions. The critical wave number $q_{c}$ is determined numerically as the threshold for long-term growth of $S$. Numerical results for $\mathcal{P}=5$ and the sinegenerated initial condition $\theta_{ \pm 1}(0)=\mp i \epsilon / 2$ are shown in Fig. 1 , trace $D$. Equation (9) underestimates these results by at most $3 \%$ for $S \leqslant 7$, and overestimates numerical results for the Kinoshita initial condition $\theta_{ \pm 1}(0)=\theta_{ \pm 1}^{(1)}$ and $\theta_{ \pm 3}(0)$ $=\theta_{ \pm 3}^{(3)}$ by the same margin. Thus, although the numerical critical wave number $q_{c}$ does depend on the relative values of the initial mode amplitudes, it is evidently confined to a

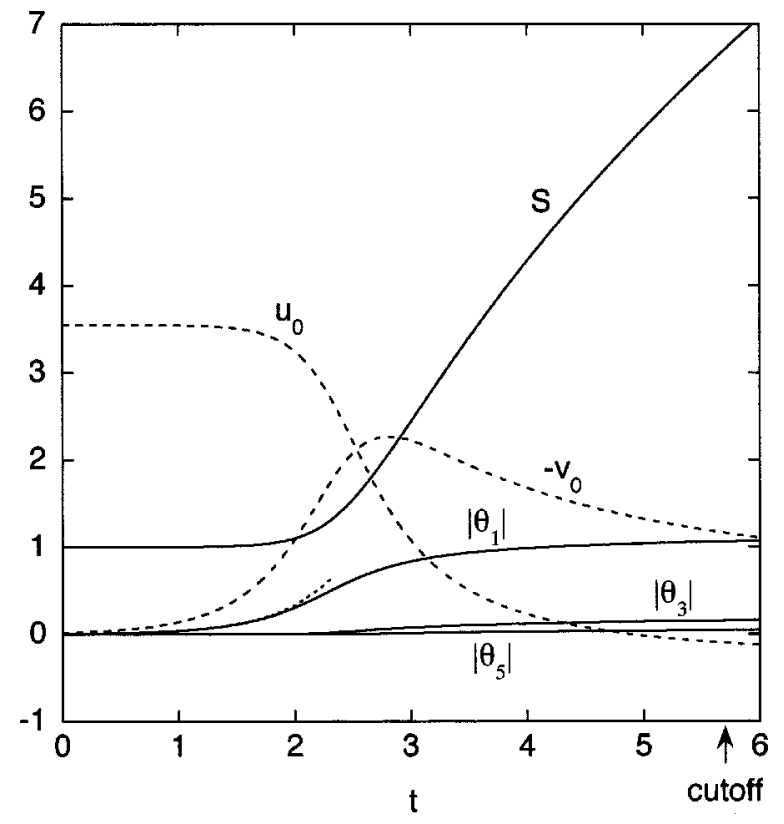

FIG. 2. Time development of the sinuosity $S$, mode amplitudes $\left|\theta_{l}\right|$, and lateral and down-valley migration rates $-v_{0}$ and $u_{0}$ of a bend apex, for an initial small-amplitude sinusoidal river centerline. Short-time results agree with linear theory, which predicts $u_{0}$ $=3.55, S=1$, and exponential growth for both $\left|\theta_{1}\right|$ (incomplete dashed trace) and $-v_{0}$. Nonlinear predictions include the peak in $-v_{0}$ at $t \approx 3$ and the negative values of $u_{0}$ near oxbow cutoff at $t$ $=5.69$, which implies that the bend apex begins migrating up-valley just before cutoff.

narrow band centered around the prediction of Eq. (9), and is bounded strictly from above by Eq. (8).

In summary, Fig. 1 illustrates our extension of research on meandering rivers into the nonlinear regime: trace $A$ gives the weakly nonlinear critical wave number representing the prior state of the art $[5,7]$; traces $B$ and $C$ give our new analytical approximations; and trace $D$ gives our exact fullynonlinear result.

To investigate all stages of growth for $q<q_{c}$ in a single simulation, we use the sine-generated initial condition with $\epsilon=0.01, q=q_{m}$, and $\mathcal{P}=5$. Figure 2 shows the resulting time development, which agrees precisely with the linear results $u_{0}=c=3.55, S=1$, and $\left|\theta_{1}\right|=(\epsilon / 2) e^{\sigma_{m} t}$ (incomplete dashed trace) at small times. Nonlinear effects above $t>2$ increase the sinuosity $S$, decrease the down-valley migration rate $u_{0}$, and introduce higher-order modes, whose amplitudes remain small compared with $\left|\theta_{1}\right|$, whose slow growth at long times is sufficient to produce surprisingly rapid growth of $S$. Remarkably, nonlinear effects eventually reverse the downvalley migration of bend apexes, which actually travel upvalley at long times approaching cutoff. The lateral apex migration rate $-v_{0}$ reaches a peak near $t=3$ and decreases slowly thereafter. Figure 3 shows the river centerline at $t$ $=2, t=3.5$, and $t=5.69$, when the centerlines of adjacent river bends meet, in a manner similar to Fig. 7 of Seminara [12]. Whereas Seminara included the $l=1$ and $l=3$ modes, our simulations include all modes through $l=33$. Figure 2 shows that $\left|\theta_{5}\right|$ reaches about $30 \%$ of $\left|\theta_{3}\right|$ at cutoff, indicat- 


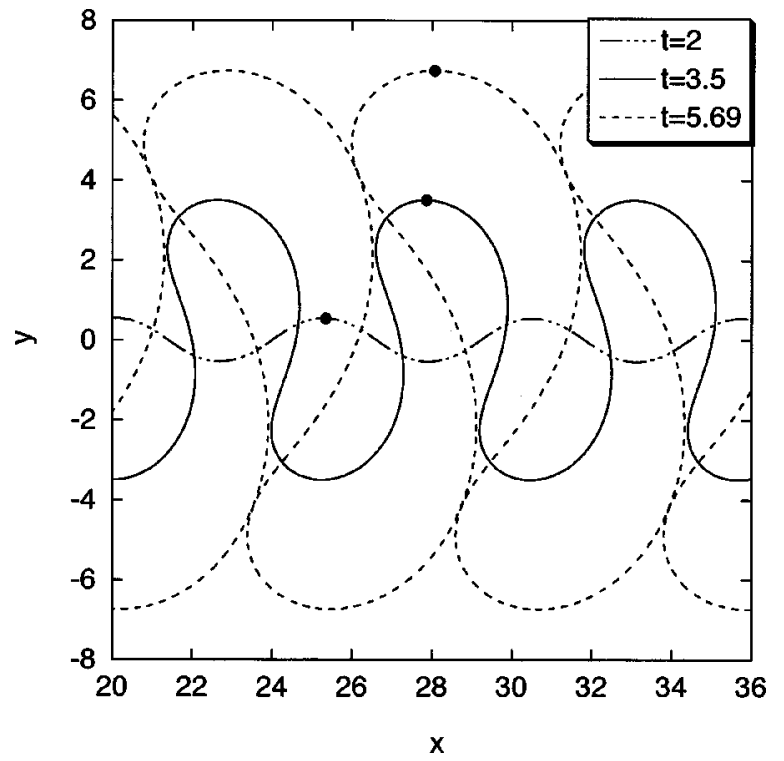

FIG. 3. River centerlines at dimensionless times $t=2,3$, and 5.69 for the simulation discussed in Fig. 2, with black dots identifying a bend apex as it migrates laterally and down-valley. Flow is from left to right.

ing that the $l=5$ mode plays a measurable role in the dynamics. The sinuosity $S=6.69$ and amplitude $2\left|\theta_{1}\right|=2.12$ $=122^{\circ}$ at $t=5.69$ are upper limits on the oxbow cutoff values because finite-width rivers will cutoff earlier. Kinoshita $(l=3)$ skewing, which causes midvalley sections of the river to travel down-valley faster than bend apexes, is evident at $t=3.5$ and $t=5.69$.
Controlled experimental tests on periodic flumes would help to determine the suitability of the model and to guide future theoretical efforts. Although nonperiodic simulations $[8,9,11,15,16]$ capture features of meandering that are seen in natural rivers, such heuristic comparisons are insufficiently precise to allow judgments of the relative merits of various models. Periodic flume experiments should be achievable in inclined tanks of sand [17] by etching the desired initial shape in an otherwise planar sand surface. A series of experiments on small-amplitude sine-generated rivers of different wavelengths might be used to identify the critical wavelength $\lambda_{c}=2 \pi D_{0} / q_{c}=2 \pi D_{0} \mathcal{P}^{-1 / 2}$ and the wavelength of maximum growth $\lambda_{m}=2 \pi D_{0} / q_{m}=2 \pi D_{0}[-1+(1$ $\left.+\mathcal{P})^{1 / 2}\right]^{-1 / 2}$ in the linear regime, both measured in conventional units, which would determine values of $D_{0}$ and $\mathcal{P}$. Using these values, direct comparison of experimental results for $\lambda_{c}=2 \pi D_{0} / q_{c}$ in the nonlinear regime could be made with the predictions summarized in Fig. 1. Experimental confirmation of the predicted peak in $-v_{0}$ and the predicted upstream migration of bend apexes at long times would also be useful. Since the model is not restricted to periodic rivers, such comparisons might increase the confidence placed on its predictions for natural rivers.

We gratefully acknowledge fruitful interactions with Gary Parker, S. F. Edwards, Alan Kerstein, and Tao Sun. This work was accomplished during a stay (B.F.E.) at the National Energy Technology Laboratory, and was funded by the Office of Fossil Energy of the U. S. Department of Energy.
[1] F. K. Lutgens and E. J. Tarbuck, Essentials of Geology (Prentice Hall, Englewood Cliffs, NJ, 1995), Chap. 9.

[2] S. Ikeda, G. Parker, and K. Sawai, J. Fluid Mech. 112, 363 (1981).

[3] B. F. Edwards and D. H. Smith (unpublished).

[4] H. Johannesson and G. Parker, River Meandering, edited by S. Ikeda and G. Parker (American Geophysical University, Washington DC, 1989), p. 181.

[5] G. Parker and E. Andrews, J. Fluid Mech. 162, 139 (1986).

[6] G. Parker, P. Diplas, and J. Akiyama, J. Hydraul. Div., Am. Soc. Civ. Eng. 109, 1323 (1983).

[7] G. Seminara and M. Tubino, J. Fluid Mech. 244, 257 (1992); P. Blondeaux and G. Seminara, ibid. 157, 449 (1985).

[8] P. Meakin, T. Sun, T. Jossang, and K. Schwarz, Physica A 233, 606 (1996); T. Sun, P. Meakin, T. Jossang, and K. Schwarz, Water Resour. Res. 32, 2937 (1996).

[9] H. H. Stolum, Science 271, 1710 (1996); Phys. Rev. E 56, 6710 (1997); Bull. Geol. Soc. Am. 110, 1485 (1998).

[10] S. M. Beck, Proceedings Rivers '83, Spec. Conf. River Me- and., ASCE, 932 (American Society of Civil Engineers, New York, 1983); A. D. Howard and T. R. Knutson, Water Resour. Res. 20, 1659 (1984).

[11] T. B. Liverpool and S. F. Edwards, Phys. Rev. Lett. 75, 3016 (1995).

[12] G. Seminara, in Nonlinear Dynamics and Pattern Formation in the Natural Environment, edited by A. Doelman and A. van Harten, Pitman Research Notes in Mathematics Series Vol. 335 (Longman, New York, 1995), pp. 269-294.

[13] G. Zolezzi, Ph.D. thesis, University of Genova, 1999 (unpublished).

[14] L. B. Leopold and M. G. Wolman, Bull. Geol. Soc. Am. 71, 769 (1960).

[15] W. B. Langbein and L. B. Leopold, U. S. Geol. Surv. Prof. Pap. 422H (1966).

[16] D. H. Smith and B. F. Edwards (unpublished).

[17] E. Stokstad, Science 287, 1912 (2000); S. A. Schumm, M. P. Mosley, and W. E. Weaver, Experimental Fluvial Geomorphology (Wiley, New York, 1987). 